\title{
A narrative review of the occurrence of posttraumatic stress responses in adolescent and young adult cancer survivors
}

This article was published in the following Dove Press journal:

Clinical Oncology in Adolescents and Young Adults

18 February 2015

Number of times this article has been viewed

\author{
Stefanie C Vuotto' \\ Katia M Perez ${ }^{2}$ \\ Kevin R Krull' \\ Tara M Brinkman' \\ 'Department of Epidemiology and \\ Cancer Control, St Jude Children's \\ Research Hospital, Memphis, \\ ${ }^{2}$ Department of Psychology and \\ Human Development, Vanderbilt \\ University, Nashville, TN, USA
}

\begin{abstract}
Adolescent and young adult cancer survivors may experience posttraumatic stress responses following cancer diagnosis or treatment. The current paper reviews 23 studies reporting the occurrence of posttraumatic stress symptoms (PTSS) and posttraumatic stress disorder (PTSD), and associated predictors of these outcomes in adolescent and young adult cancer survivors. Results indicate considerable variability among prevalence estimates of PTSD (0\%-34.8\%) and PTSS (4.4\%-78\%). Measurement inconsistencies limiting the ascertainment of reliable prevalence and risk estimates are discussed in the context of the reviewed literature. Specifically, differences in assessment measures utilized, the timing of assessment relative to diagnosis, the criteria used to define the outcome, and identification of the precipitating traumatic event may account for discrepancies in prevalence and risk estimates across studies. The application of specific PTSD diagnostic criteria to a survivorship population is discussed. Empirically supported interventions utilizing cognitive behavioral therapy approaches for the treatment of PTSS in adolescent and young adult cancer survivors are identified.
\end{abstract}

Keywords: posttraumatic stress, adolescent and young adult, cancer survivors

\section{Introduction}

Cancer is the leading cause of disease-related death among children in the United States, with over 15,000 children $<19$ years of age diagnosed with cancer each year. ${ }^{1}$ Climbing incidence combined with increasing 5-year survival rates, presently at roughly $80 \%,^{2,3}$ have contributed to a growing number of pediatric cancer survivors. Currently, it is estimated that there are approximately $400,000^{4}$ pediatric cancer survivors living in the United States with $70 \%$ of these survivors aged 20 years or older. ${ }^{1}$ Approximately 70,000 adolescents and young adults (AYA) 15-39 years of age are diagnosed with cancer in a single year; roughly six times the number of cases diagnosed in children $<14$ years of age. ${ }^{5}$ Less than half of the 24 most common AYA cancer diagnoses have a 5-year survival rate exceeding $80 \% .{ }^{6}$ Compared with survival trends among pediatric and older cancer populations, there has been limited progress in improving the survival rates of AYA cancers in recent decades.

Research investigating the psychosocial sequelae of pediatric and AYA cancer suggests survivors' psychological adjustment may be adversely impacted. Given that trauma and chronic stress, such as life-threatening illnesses and their sequelae, have been found to influence psychological adjustment, recent attention has focused on the occurrence and development of traumatic stress responses in young cancer survivors. With the broadening of diagnostic criteria for posttraumatic stress disorder (PTSD) in the fourth edition of the Diagnostic and Statistical Manual of Mental Disorders
Correspondence: Kevin R Krul

262 Danny Thomas Place, MS 735,

Memphis, TN 38105, USA

$\mathrm{Tel}+\mid$ 90I $595589 \mid$

Fax +l 90I 5955845

Email kevin.krull@stjude.org
Clinical Oncology in Adolescents and Young Adults 2015:5 19-33 
(DSM-IV) to include "being diagnosed with a life-threatening illness", ${ }^{7}$ studies examining the prevalence of PTSD and posttraumatic stress symptoms (PTSS) among cancer patients and survivors have emerged. The objectives of this review include the following: 1) to report prevalence estimates of PTSD and PTSS in AYA cancer survivors; 2) to review the established risk factors believed to precipitate PTSD and PTSS in AYA cancer survivors; 3 ) to discuss the methodological challenges associated with ascertaining prevalence estimates of PTSD and PTSS in AYA cancer survivors; and 4) to comment on the state of evidence-based treatment and intervention in the affected population.

\section{The AYA survivor population}

AYA cancer survivors make up a heterogeneous population comprised of subgroups that vary with respect to age at diagnosis, treatment, and developmental features. There are two general cohorts included in the definition of AYA survivors: those diagnosed with cancer as children or adolescents (ie, treated on the pediatric side) who have survived into adolescence/young adulthood; and those diagnosed as AYA. The National Cancer Institute defines AYA cancer patients as those diagnosed with cancer between the ages of 15 years and 39 years. $^{8}$

Because adolescence and young adulthood are life stages defined by unique developmental challenges and vulnerabilities, AYA survivors may have different cancer and survivorship experiences depending on age at diagnosis. For example, treatment may have occurred at pediatric or adult medical centers, long-term follow-up care may or may not be available, and psychological development may have been disrupted at different phases of development. Like their healthy peers, AYA survivors must develop a sense of self in order to continue along a healthy developmental trajectory and master future developmental milestones. An interruptive adverse life event such as a cancer diagnosis is likely to demand the attention and focus of resources that might have been directed elsewhere, including developing a positive body image and sexual identity and establishing autonomy from parents. Disrupted developmental milestones will vary depending on age, with adolescent milestones characterized by the transition from dependence to autonomy, and young adult milestones typically related to establishing financial independence, forming intimate relationships, and family planning. Unlike survivors diagnosed as young children, survivors diagnosed as AYAs have the cognitive capacity to understand the implications of a life-threatening illness and are forced to confront their own mortality during a life period typically marked by feelings of invincibility. ${ }^{9}$ Unresolved conflicts related to personal identity, life meaning, or achievement may resurface after the completion of treatment and manifest in the form of persistent psychological distress or traumatic stress responses.

\section{Syndrome versus symptoms: PTSD and PTSS}

Currently, the most widely published and clinically utilized definition of PTSD comes from the DSM-IV, which classifies PTSD as an anxiety disorder and requires that the disorder follow exposure to a traumatic event (through witnessing or experiencing) which threatened the life or bodily integrity of the self or other.

In the DSM-IV, PTSD is defined by three symptom clusters (re-experiencing, avoidance, and hyperarousal) which, when combined, produce clinical levels of distress and/or impairment for at least 1 month following the traumatic event (Table 1). Using the DSM-IV criteria, the overall lifetime prevalence of PTSD is approximately $8 \%$ among adults in the United States and the past year (12-month) prevalence is estimated at $3.5 \%$, with higher rates consistently found among women. ${ }^{7}$ Reported PTSD rates among American adolescents vary. Data from the National Survey of Adolescents, which used a household probability sample of 4,023 adolescents aged 12-17 years, reported the 6-month prevalence as $3.7 \%$ for boys and $6.3 \%$ for girls. ${ }^{10}$ Data from the National Comorbidity Survey Replication-Adolescent Supplement, which used a nationally representative sample of more than 10,000 adolescents between the ages of 13 years and 18 years, indicated that the lifetime prevalence rate for PTSD is 5\%, with higher rates among females. ${ }^{11}$ Significantly higher PTSD prevalence rates have been reported among certain at-risk populations, such as service members deployed in Operation Enduring Freedom and in Operation Iraqi Freedom $(13.8 \%),{ }^{12}$ survivors of human-made/technological disasters $(30 \%-60 \%),{ }^{13}$ survivors of natural disasters $(5 \%-60 \%),{ }^{13}$ and cancer patients and survivors $(3 \%-35 \%) .{ }^{14}$

The provision of a developmental framework for considering the diagnosis of PTSD did not formally emerge until the fifth edition of the DSM (DSM-5). ${ }^{15}$ In DSM-5, the inclusion of a second set of diagnostic criteria for children 6 years of age and younger, as well as text notes for symptom expression in children older than 6 years, has helped clarify the diagnosis of PTSD in children (Table 3). New DSM-5 text revisions regarding the development and course of the disorder throughout childhood, adolescence, and young adulthood provide clinically useful age guidelines. For example, 
Table I DSM-IV: posttraumatic stress disorder criteria

\begin{tabular}{|c|c|c|}
\hline DSM-IV criteria & Operationalization & Considerations for AYA cancer survivors \\
\hline \multicolumn{3}{|l|}{ Criterion A } \\
\hline Exposure to traumatic event threatening & Response characterized by intense fear, & Cancer not a discrete event \\
\hline death or serious injury of self or others & helplessness, or horror & Risk of focusing illusion \\
\hline \multicolumn{3}{|l|}{ Criterion B } \\
\hline Re-experiencing (one or more symptom[s] & Intrusive recollections & Well-founded fears about relapse/future health \\
\hline \multirow[t]{4}{*}{ required) } & Distressing dreams & \\
\hline & Recurring or reliving the event & \\
\hline & Internal/external cue(s) triggering psychological & \\
\hline & distress or physiological reactivity & \\
\hline \multicolumn{3}{|l|}{ Criterion $\mathbf{C}$} \\
\hline \multirow{6}{*}{$\begin{array}{l}\text { Avoidance (three or more symptom[s] } \\
\text { required) }\end{array}$} & Effortful avoidance of thoughts, feelings, & Long-term care/late effects make avoidance \\
\hline & or anything associated with the trauma & difficult \\
\hline & Inability to recall aspects of the trauma & Cognitive impairment \\
\hline & Social withdrawal, diminished interest in activities & Psychosocial sequelae \\
\hline & Emotional numbing & Possibility of recurrence \\
\hline & Sense of a foreshortened future & \\
\hline \multicolumn{3}{|l|}{ Criterion D } \\
\hline Arousal (two or more symptom[s] & Difficulty falling or staying asleep & Neurocognitive late effects \\
\hline \multirow[t]{4}{*}{ required) } & Irritability or outbursts of anger & Sleep-related late effects \\
\hline & Concentration difficulty & \\
\hline & Hypervigilance & \\
\hline & Exaggerated startle response & \\
\hline \multicolumn{3}{|l|}{ Criterion $\mathbf{E}$} \\
\hline Duration of disturbance & Symptoms persist more than I month & Late effects typically persist beyond 30 days \\
\hline \multicolumn{3}{|l|}{ Criterion F } \\
\hline Clinically significant distress & & Cancer therapy late effects may impact \\
\hline or functional impairment & & functioning \\
\hline
\end{tabular}

Abbreviations: DSM-IV, Diagnostic and Statistical Manual of Mental Disorders, fourth edition; AYA, adolescent and young adult.

symptom expression shifts across developmental stages, with adolescents likely to exhibit feelings of social undesirability and/or irritable, aggressive, or reckless behavior, and young adults likely to present with hyperarousal, avoidance, and/ or negative cognitions and mood..$^{15}$

It is possible for an individual to experience one or more of the symptoms associated with PTSD without having the full disorder. These symptoms, known as posttraumatic stress symptoms (PTSS), provide a more continuous measure of the pathological traumatic stress response and are often used as clinical indicators for intervention and treatment. PTSS often cluster together but do not require the prolonged duration or functional impairment criteria needed for a full diagnosis of PTSD, making PTSS a more commonly observed trauma response. ${ }^{16}$ Though PTSS lack clinical diagnostic criteria, their clinical utility should not be underestimated. Individuals experiencing PTSS who do not meet the threshold for PTSD may qualify for a diagnosis of acute stress disorder or adjustment disorder, and may be considered candidates for further clinical evaluation, monitoring, or treatment. In research contexts, the PTSS construct is used far more broadly, usually encompassing any posttraumatic symptom that does not result in a diagnosis of PTSD. The broad interpretation of PTSS in research is demonstrated in the cancer survivorship literature, with reported rates of PTSS ranging from $15 \%-88 \% .{ }^{17}$ Despite this variability, the widespread investigation of PTSS in cancer survivorship research has yielded valuable epidemiologic, qualitative, and clinical information that further elucidates the adjustment experience of young cancer survivors.

\section{Review of the literature}

A review of the literature was conducted using PubMed and PsycINFO databases, which were searched using the following key terms: posttraumatic stress; posttraumatic stress disorder; adolescent; young adult; cancer; and cancer survivor. Studies meeting the following inclusion criteria were included in the review: 1) published within the last 20 years; 2) had sample sizes of at least 20 individuals; 3 ) conducted in the United States; 4) used DSM criteria in the assessment of PTSD and psychometrically-sound measures in the assessment of PTSS; 5) reported the prevalence of PTSD and/or PTSS; and 6) used samples of childhood cancer survivors in adolescence/ young adulthood and/or survivors of AYA cancer. Criterion 6 was applied to examine the potential differences in prevalence between cases where initial diagnosis was thought to 
be directly disruptive to AYA development and cases where the impact was thought to endure into AYA survivor years. The rationale for including both populations was also based on the fact that common AYA malignancies have considerable overlap with both pediatric and adult cancers. ${ }^{8}$ In accord with the aims of this paper, the methodological characteristics of the 23 reviewed studies are presented, followed by a description of the overall occurrence of posttraumatic stress responses among AYA cancer survivors. An overview of the studies reviewed can be found in Table 2 .

\section{Study methodologies}

Of the 23 studies reviewed, five studies focused on adolescent cancer survivors, ${ }^{18-22}$ ten focused on adult (18 years and older) survivors of pediatric or AYA cancers, ${ }^{16-17,23-30}$ and eight focused on both. ${ }^{31-38}$ The reported ages of participating survivors ranged from 7 years ${ }^{21}$ to 71 years. ${ }^{29}$ With regard to the heterogeneity of diagnoses, the majority of studies examined survivors of all cancers, with seven studies excluding central nervous system malignancies, ${ }^{17,19,22,25,31,34,38}$ and only one focusing on Hodgkin's lymphoma survivors. ${ }^{29}$ The majority (number $[\mathrm{n}]=14$ ) of studies examined both PTSS and PTSD outcomes; five assessed PTSD alone ${ }^{16,18,25,36,37}$ and four evaluated PTSS alone..$^{22,27,34,38}$ Sample sizes ranged from 23-6,542, with ten samples of less than 100, ${ }^{17,19,23-25,30-32,36}$ eleven samples between 100 and $350,{ }^{18,20,22,26,29,33-35,37,38}$ and two samples exceeding 6,500..$^{16,28}$ Most studies employed a cross-sectional design, with only four studies using a longitudinal design. ${ }^{17,35,37,38}$ Only nine studies included a comparison or control group. . $^{16,17,21,28,29,31,34,36,37}$

Seven self-report measures, including the Adolescent Dissociative Experiences Scale-II (A-DES), ${ }^{39}$ Posttraumatic Stress Diagnostic Scale (PDS), ${ }^{40}$ University of California at Los Angeles Posttraumatic Stress Disorder Index (PTSDI), ${ }^{41}$ Posttraumatic Stress Checklist-Civilian Version (PCL-C), ${ }^{42}$ Child Posttraumatic Stress Reaction Index - Revision 2 (CPTS-RI), ${ }^{43}$ Trauma Symptom Checklist for Children Alternate version (TSCC-A), ${ }^{44}$ Posttraumatic Stress Disorder Reaction Index (PTSD-RI), ${ }^{41}$ and one interview (Impact of Traumatic Stressors Interview Schedule [ITSIS] $)^{33}$ were used to measure PTSS. Three of these measures (PDS, PTSD-RI, and PTSDI) were also used to indicate "likely" cases of PTSD when scores fell in the clinically significant range. Five assessment instruments, all structured and semistructured interviews, were used to assess PTSD: Kiddie-Schedule for Affective Disorders and Schizophrenia-Epidemiologic Version-5 (K-SADS-E-5); ${ }^{45}$ Structured Clinical Interview for DSM-IV (SCID); ${ }^{46}$ Clinician-Administered PTSD Scale for Children and Adolescents (CAPS-CA); ${ }^{47}$ Structured Interview for PTSD (SI-PTSD); ${ }^{48}$ and Diagnostic Interview Schedule for Children (DISC 2.3). ${ }^{49}$ Psychological distress and functional impairment were assessed using the Brief Symptom Inventory - 18 (BSI-18); ${ }^{50}$ Impact of Event Scale (IES) ${ }^{51}$ Medical Outcomes Study 36-Item Short-Form Health Survey (SF-36); 52 Short-Form Health Status questionnaire (SF-12); ${ }^{53}$ and Global Assessment of Functioning scale (GAF). ${ }^{54}$

The studies reviewed in this paper often identified the temporal relation between diagnosis/treatment and assessment of PTSD/PTSS (eg, how much time elapsed since diagnosis or treatment at time of assessment) rather than explicating whether PTSD/PTSS was linked to the cancer experience. At least half $(n=12)$ of the studies did not address whether or not PTSD symptoms were related to cancer, while three reported the number of cases in which the self-reported traumatic event related to the survivor's cancer experience, ${ }^{17,29,36}$ four examined cancer-induced PTSD by having subjects respond to items on the basis of their cancer experience or adapting items to reflect the cancer experience (eg, by replacing the word "trauma" with "cancer experience"), ${ }^{18,21,31,33}$ three studies assumed that all participants were exposed to a traumatic event because the cancer diagnosis and treatment would constitute a traumatic event, ${ }^{16,32,35}$ and one study measured PTSS 2 months after a clinic visit to limit health factors from acting as traumatic stress triggers. ${ }^{38}$ Given the different methodological approaches used toward focusing survivors on cancer as a precipitating traumatic event, ${ }^{55}$ it is important that cancer-induced PTSD prevalence estimates are interpreted with this in mind.

As with most trauma research, it is important to consider the extent to which the participation of potentially eligible survivors affects prevalence estimates. Based on 21 studies reporting participation, survivor participation ranged from $38 \%-89 \% .^{17,25}$ Research procedures requiring participants to engage in emotionally burdening interviews may cause eligible survivors to resist revisiting potentially traumatic life events and may explain nonparticipation or attrition. Nonparticipation introduces potential selection bias and may influence the precision of prevalence estimates, the magnitude of observed risk, and the generalizability of study findings.

\section{Prevalence}

The occurrence of PTSD was assessed in 19 studies and ranged from $0 \%{ }^{31}$ to $34.8 \% .{ }^{36}$ This prevalence range was narrowed to $0.77 \%-20.5 \%$ when three studies that used DSM-III criteria $^{31,32,36}$ and three studies that measured the occurrence of 
"likely" PTSD, as determined by clinically significant PTSS scores and/or which did not include functional impairment, were excluded..$^{21,24,35}$ Further excluding one study in which no survivors reported cancer-related traumatic events, ${ }^{17}$ the prevalence ranged from $8 \%{ }^{20}$ to $20.5 \% .^{23}$

The prevalence of PTSS was assessed in 18 studies. Two studies reported the prevalence of intrusion and avoidance symptoms among young adult cancer survivors, as measured by the IES; clinically significant avoidance symptoms ranged from $6 \%-16.7 \%$, while clinically significant intrusion symptoms ranged from $4.4 \%-9 \%{ }^{23,26}$ Five studies reported the prevalence of the three PTSS clusters identified in the DSM-IV using the PTSD-RI, PTSDI, and PCL-C: $40 \%{ }^{24}$ to $75.3 \%{ }^{26}$ of young adults, $23.6^{38}$ to $73.3 \%{ }^{20}$ of adolescents, and $50 \%$ of children and adolescents reported re-experiencing symptoms; $14.7 \%{ }^{33}$ to $25.8 \%{ }^{26}$ of young adults, $14.3 \%{ }^{38}$ to $16.1 \%{ }^{20}$ of adolescents, and $16.7 \%^{20}$ of children and adolescents experienced avoidance symptoms; and $31 \%{ }^{33}$ to $55.5 \%{ }^{24}$ of young adults, $20 \%{ }^{38}$ to $41.3 \%^{20}$ of adolescents, and $28.8 \%{ }^{20}$ of children and adolescents reported experiencing arousal symptoms. Moderate to severe PTSS scores were reported in $7.7 \%^{23}$ to $36 \%^{31}$ of AYA survivors when using the PTSD-RI and $39 \%-44 \%{ }^{35}$ (depending on the time at assessment) of AYA survivors when using the PSD. PTSS that amounted to "partial PTSD" (eg, endorsing at least one, but not all, PTSD symptom clusters) were observed in $4.8 \%$ to $35.2 \%{ }^{29}$ of participants when the symptom threshold was two, and $7 \%{ }^{17}$ to $78 \%{ }^{32}$ when the symptom threshold was one. Clinically significant PTSS scores on the PTSDI, PCL-C, and PDS (ie, exceeding clinical cutoff scores and/or meeting all three symptom cluster criteria without functional impairment) ranged from $4.1 \%{ }^{21}$ to $13.3 \%{ }^{27}$ among survivors of all ages, and they ranged from $7.5 \%{ }^{28}$ to $13.3 \%{ }^{27}$ among AYA survivors. Two studies reported no significant difference between AYA cancer survivors and comparison group levels of PTSS. ${ }^{21,34}$

\section{Factors associated with PTSS/PTSD}

A number of static risk factors have been linked to the occurrence and development of posttraumatic stress responses in AYA cancer survivors (Table 4). While these fixed, unalterable factors may not be amenable to treatment or intervention, they may be utilized by clinicians in primary preventive efforts. Female sex has generally been associated with risk for cancer-related PTSS/PTSD, although the magnitude and consistency of this association is unclear. While one study identified female sex as a significant contributor to PTSS $(\beta=0.13),{ }^{22}$ another reported a negligible odds ratio $(\mathrm{OR})$ for female sex $(\mathrm{OR}=0.17),{ }^{24}$ and one-third found no association. ${ }^{25}$ Individuals diagnosed at older ages have been reported to exhibit more PTSS. Diagnosis during adolescence has been associated with a higher frequency of PTSD symptoms when compared with children diagnosed at school age, ${ }^{34}$ and young adults have demonstrated greater rates of $\mathrm{PTSD}^{16}$ and higher scores on the PTSD-RI and IES than child and adolescent survivors. ${ }^{23}$ Several studies have documented positive associations between medical late effects and posttraumatic stress responses $\left(r=0.49 ;{ }^{17}\right.$ $r=0.32 ;{ }^{31}$ and $\left.r=0.43\right),{ }^{35}$ as well as perceived impact of late effects and PTSD $(t[179]=-2.85),{ }^{26}$ while other research found no association. ${ }^{23}$ Greater treatment intensity has been associated with the risk of PTSD (OR $=1.36$; 95\% confidence interval [CL]: $1.06-1.74)^{16}$ and anxiety and avoidance, ${ }^{34}$ although one study found no significant association between chart-derived ratings of treatment intensity and PTSS. ${ }^{23}$ Lower educational level, ${ }^{16}$ unemployment, ${ }^{35}$ having a history of stressful events, ${ }^{22}$ and having experienced a relapse or recurrence ${ }^{21}$ also emerged as salient predictors of PTSS/ PTSD. It should be noted that for long-term follow-up studies, lower education and unemployment may actually be a consequence of PTSS/PTSD symptoms rather than a predictor; prospective longitudinal research would be needed to confirm causal associations. Other treatment and demographic factors, such as time since treatment and age at diagnosis, were not consistently associated with PTSS. The presence of a repressive adaptation style was associated with lower levels of PTSS and PTSD, ${ }^{19,21,32}$ while trait and general anxiety were associated with greater PTSS. ${ }^{22,23}$

The more malleable, dynamic predictors of posttraumatic stress are likely to be the target of future intervention. Results of several studies suggest that perceived severity of cancer diagnosis/life threat and other subjective appraisals are related to posttraumatic stress. In a model predicting PTSS, survivor appraisal of life threat and treatment intensity significantly contributed to PTSS $(\beta=0.22) .{ }^{22}$ Another study found that young adult survivors with PTSD were more likely to self-report higher current life threat and rate their cancer treatment as more intense. ${ }^{23}$ Though perceptions are often functions of personality, perceptions are amenable to change, making them ideal targets for treatment and intervention. Delayed progress toward developmental goals (eg, personal, educational, family/relationship goals) was associated with increased risk for PTSD/PTSS in a young adult sample of survivors $(t[169]=5.32) \cdot{ }^{26}$ Lack of family/social support was found to contribute to the occurrence of PTSS/PTSD as well; one study of young adult survivors found that living alone 


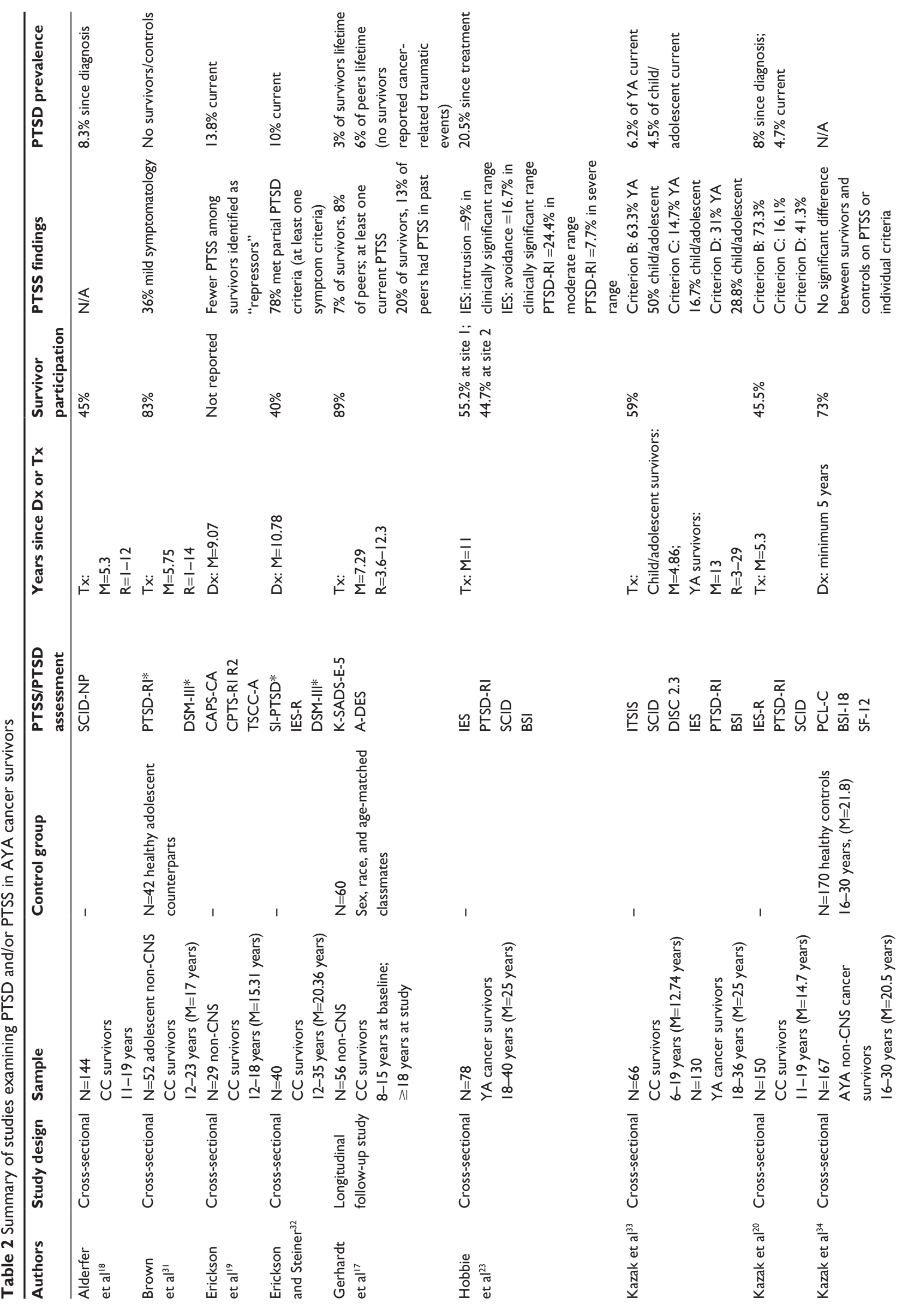




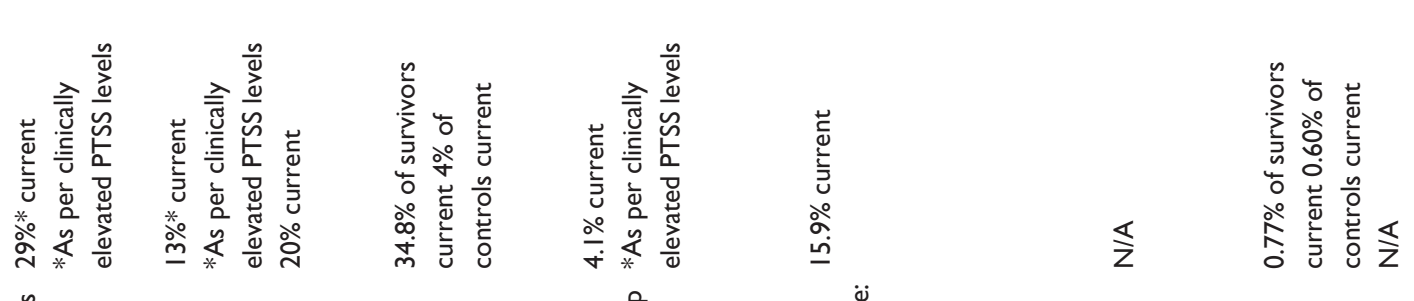

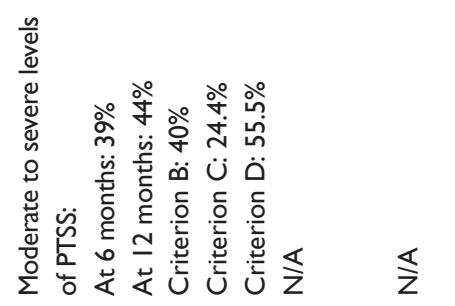

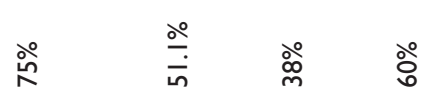

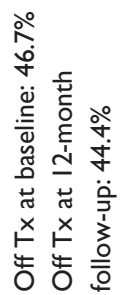

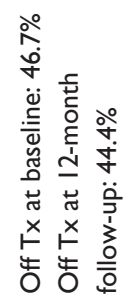

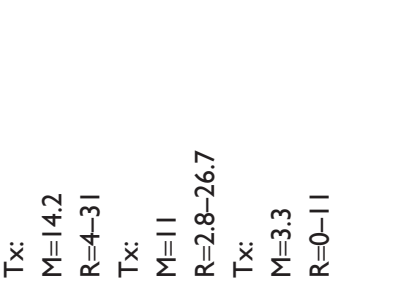

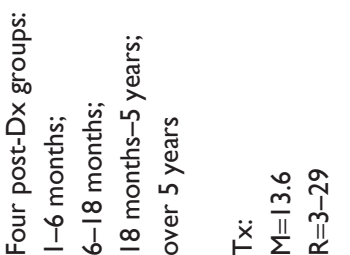

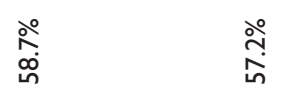

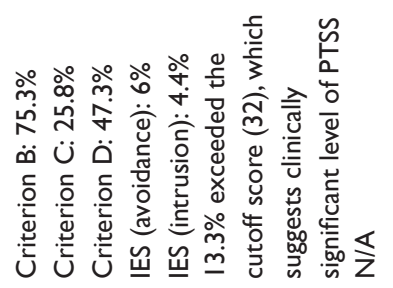

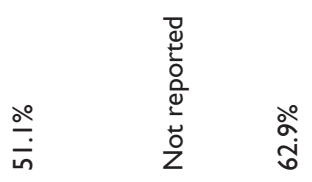

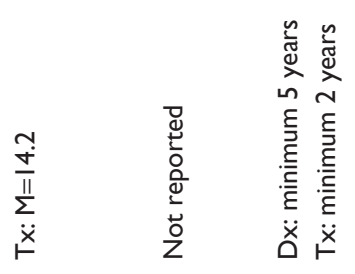

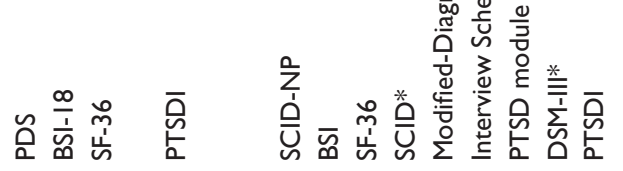

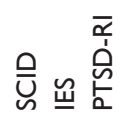

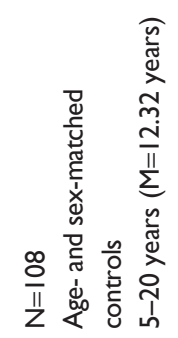

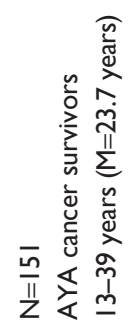
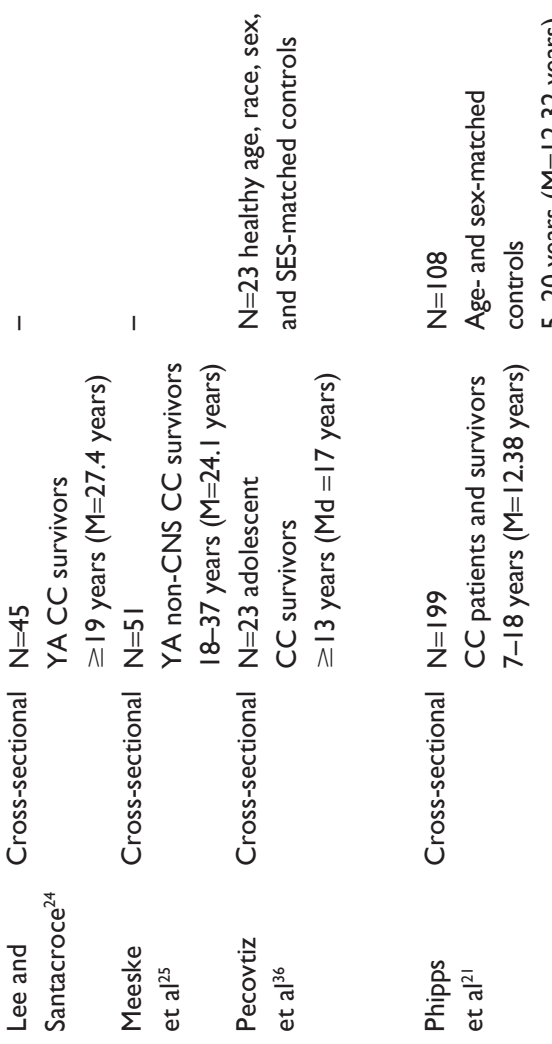

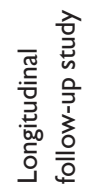

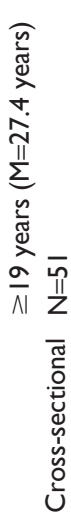
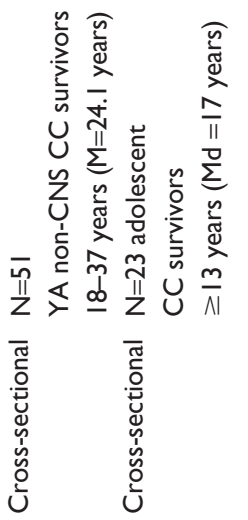

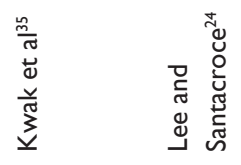

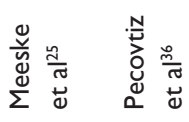

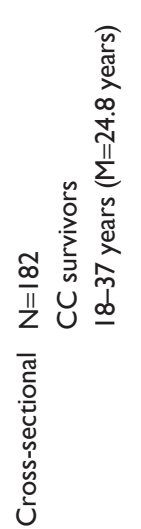

竞 苞
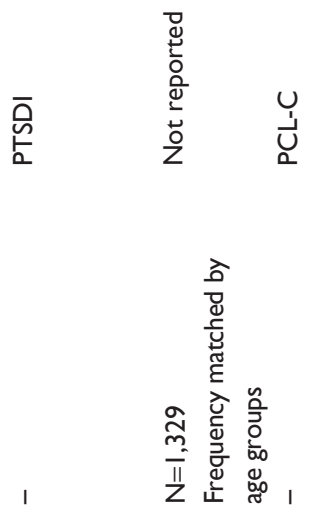


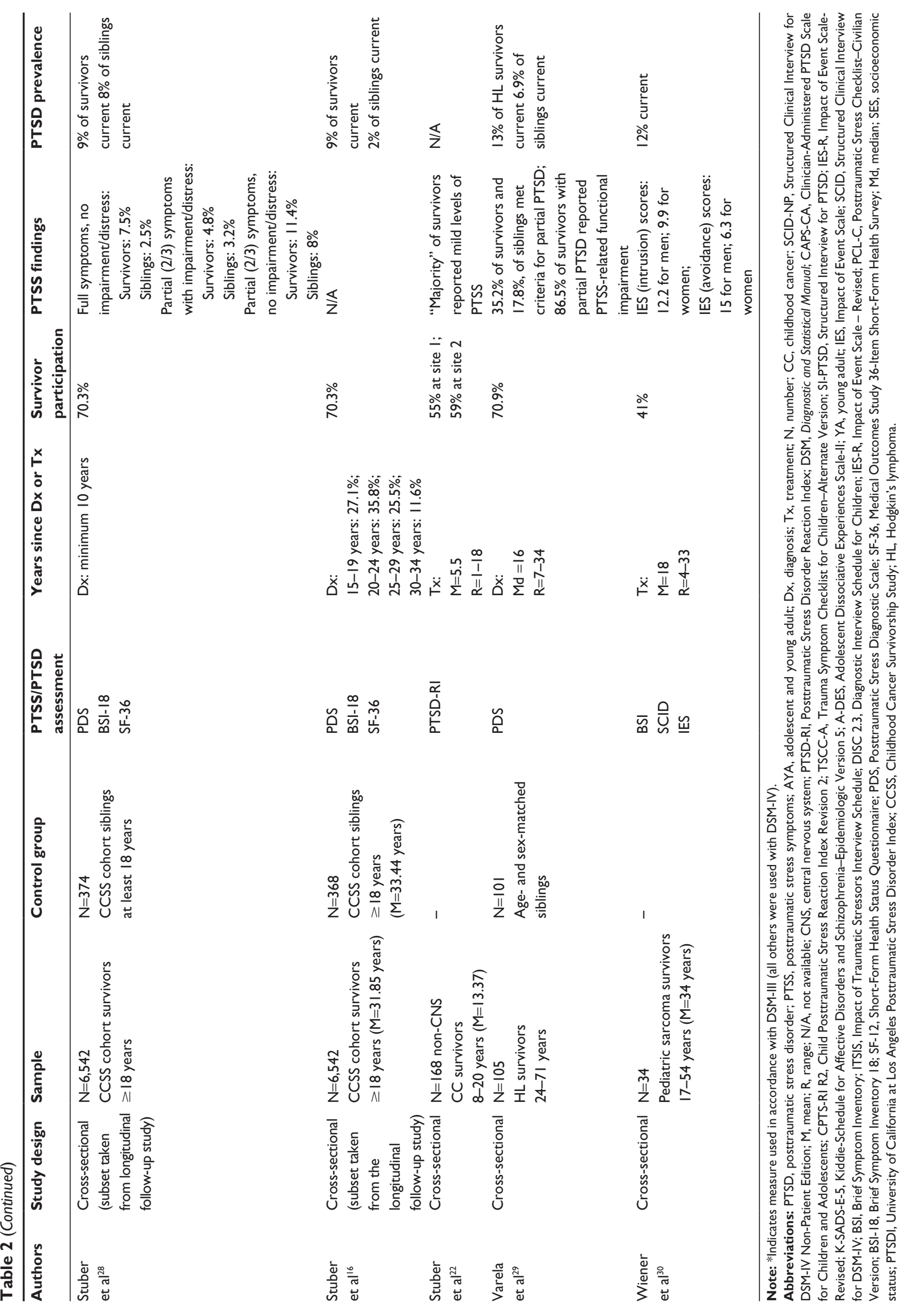


Table 3 DSM-5: posttraumatic stress disorder criteria

\begin{tabular}{lcc}
\hline DSM-5 criteria & Operationalization & $\begin{array}{c}\text { Considerations for AYA cancer } \\
\text { survivors }\end{array}$ \\
\hline
\end{tabular}

\section{Criterion A}

Exposure to traumatic event threatening death or serious injury of self or others Criterion B Intrusion (one or more symptom[s] required)

\section{Criterion C}

Avoidance (one or more symptom[s] required)

\section{Criterion D}

Negative alterations in cognition and mood (two or more symptom[s] required)

\section{Criterion E}

Alterations in arousal and reactivity (two or more symptom[s] required)

\section{Criterion F}

Duration of disturbance

\section{Criterion G}

Functional impairment

\section{Criterion $\mathbf{H}$}

Exclusion
Direct exposure; witnessing event; learning that event occurred to close other; experiencing repeated exposure to details of event

Intrusive recollections

Repetitive play with event-related themes*

Distressing dreams

Frightening dreams without recognizable content*

Flashbacks/recurring of the event

Trauma-specific reenactment in play*

Internal/external cue(s) triggering

Psychological distress, or

Physiological reactivity

Effortful avoidance of memories, thoughts, feelings, about event Effortful avoidance of external reminders about event

Inability to remember aspect of event

Persistent negative beliefs about self, other, the world

Persistent distorted cognitions about cause/consequence

of event; blame of self or others

Persistent negative emotional state

Diminished interest in activities

Feelings of detachment

Persistent inability to experience positive emotions

Irritability or outbursts of anger

Reckless/self-destructive behavior

Hypervigilance

Exaggerated startle response

Concentration difficulty

Difficulty falling or staying asleep

Symptoms persist more than I month

Significant distress or impairment in social, occupational, or other important areas of functioning

Disturbance not attributable to physiological effects of substance or medical condition
Cancer not a discrete event

Risk of focusing illusion

Well-founded fears about relapse/ future health

Long-term care/late effects make avoidance difficult

Cognitive impairment Possibility of recurrence Psychosocial sequelae/elevated risk of depression

Neurocognitive late effects Sleep-related late effects

Late effects typically persist beyond 30 days

Cancer therapy late effects may impact functioning

Psychosocial, neurocognitive symptoms potentially attributable to cancer therapy-related late effects

Note: *In children

Abbreviations: DSM-5, Diagnostic and Statistical Manual of Mental Disorders, fifth edition; AYA, adolescent and young adult.

was correlated with PTSS and PTSD, providing support for the relationship between social support and posttraumatic stress ${ }^{24}$ another study found that social support significantly predicted PTSS $(\beta=0.17) .{ }^{22}$ Other psychosocial factors such as psychological distress, quality of life, and family functioning have been strongly associated with posttraumatic stress responses. Specifically, families described as chaotic ${ }^{36}$ or having difficulty with problem solving, affective responsiveness, and affective involvement ${ }^{18}$ were associated with PTSD among AYA cancer survivors.
In terms of relational risk factors, exposure to PTSD in the home may put survivors at greater risk for the development of PTSS. Parents with anxiety or families consumed with cancer-related fears may influence the extent to which survivors experience and/or express distress symptoms. For example, one study reported that AYA cancer survivors with mothers diagnosed with PTSD were seven times more likely to develop PTSD themselves. ${ }^{36}$ Intervention efforts directed at reducing posttraumatic responses among young cancer survivors should include family systems models. 
Table 4 Risk factors associated with PTSD/PTSS in AYA cancer survivors

\begin{tabular}{lll}
\hline Static & Dynamic & Relational \\
\hline Female sex & $\begin{array}{l}\text { Perceived severity } \\
\text { of cancer diagnosis/life } \\
\text { threat }\end{array}$ & $\begin{array}{l}\text { Exposure to PTSD } \\
\text { in the home/having } \\
\text { a parent with PTSD }\end{array}$ \\
$\begin{array}{l}\text { Diagnosis during } \\
\text { adolescence }\end{array}$ & $\begin{array}{l}\text { Perceived severity } \\
\text { of treatment intensity } \\
\text { Medical late effects }\end{array}$ & $\begin{array}{l}\text { Perceived severity } \\
\text { of late effects } \\
\text { Delayed progress toward } \\
\text { developmental goals } \\
\text { Lack of family/social }\end{array}$ \\
Treatment intensity \\
$\begin{array}{l}\text { Lower educational } \\
\text { level }\end{array}$ \\
$\begin{array}{l}\text { Unemployment } \\
\text { Increased psychological } \\
\text { distress } \\
\text { Having a history } \\
\text { of stressful events }\end{array}$ \\
$\begin{array}{l}\text { Raving experienced a } \\
\text { relapse or recurrence }\end{array}$ \\
$\begin{array}{l}\text { Trait and general } \\
\text { anxiety }\end{array}$
\end{tabular}

Abbreviations: PTSD, posttraumatic stress disorder; PTSS, posttraumatic stress symptoms; AYA, adolescent and young adult.

\section{Discussion}

AYA survivors of cancer face a number of typical developmental challenges in the context of exposure to a potentially traumatic event. A significant proportion of AYA survivors report experiencing symptoms of posttraumatic stress with elevated risk observed among survivors who are female, ${ }^{22}$ those who develop medical late effects, ${ }^{17,31,35}$ those who achieve a lower socioeconomic status, ${ }^{16,35}$ and those who experience greater family discord. ${ }^{36,54}$ Importantly, our ability to report reliable prevalence estimates of PTSS and elucidate risk factors is hindered by several methodological factors including: 1) the definition and measurement of PTSD/PTSS; 2 ) the assumption of cancer as a traumatic event; and 3) the application of diagnostic symptoms to AYA cancer survivors.

\section{Issues of application, definition, and measurement}

Discrepancies in the prevalence of reported posttraumatic stress responses (namely, PTSD and PTSS) in young cancer survivors may be, in part, attributed to challenges associated with their definition and assessment. Beyond measurement difficulties, the appropriateness of applying the posttraumatic stress model to child and AYA posttraumatic stress reactions has been questioned. ${ }^{56}$ Specifically, the nature of the cancer experience - ongoing, ever evolving, and lacking clear, definitive termination - suggests that the stress reactions of survivors are more appropriately characterized as traumatic and normative responses, rather than posttraumatic, pathological ones. ${ }^{57}$ However, if it were the case that posttraumatic stress reactions were overpathologized, the occurrence of PTSD among cancer survivors would be far more common than is currently indicated. ${ }^{58}$ That only a fraction of survivors report PTSS suggests that these posttraumatic stress reactions reflect an underlying predisposition to pathological responses. Contextualizing these responses within the posttraumatic stress model and PTSD taxonomy provides survivors and mental health care providers with a framework for recognizing and treating PTSS and PTSD.

The epidemiology of posttraumatic stress in the AYA survivor population is subject to the outcome definition used. ${ }^{28}$ Unfortunately, there is a considerable degree of inconsistency in this regard, with studies using a variety of operational definitions for PTSD, thus leading to potential misclassification of the outcome and potential upward or downward biased estimates. For example, some studies may use any combination of symptom quantity, frequency, or severity in measuring posttraumatic stress, while others may employ the more formal DSM-IV PTSD diagnostic criteria, either with or without functional impairment. In a study examining the prevalence of PTSD in childhood cancer survivors and their siblings (aged $\geq 18$ years), Stuber and colleagues ${ }^{28}$ found that prevalence varied depending on how PTSD was operationalized. The prevalence, predictors, and functional impact of posttraumatic stress are likely to be affected by the varying definitions of PTSD, which limits direct comparison across studies.

Methodological concerns are not limited to measurement inconsistency. PTSS expression, diagnostic criteria, and assessment instruments have not been well validated or extensively studied among child and adolescent populations, despite developmental stage-specific diagnostic criteria. The questionable validity of PTSD instruments for use with adolescents is of concern, as most of these measures have been adapted from adult versions and validated on adult populations. ${ }^{59}$ Further, few assessment instruments have been developed within the cancer survivorship framework or validated within a survivorship population. ${ }^{57,60}$

\section{The Al problem: cancer as trauma}

The term "traumatic stressor" has undergone several revisions with regard to its conceptual and operational definition in the DSM. As previously stated, the definition most widely used in recent publications and practice comes from the DSM-IV, which requires that an individual with PTSD should have been exposed to a traumatic event in which they "experienced, 
witnessed, or were confronted with an event(s) that involved actual or threatened death or serious injury, or a threat to the physical integrity of self or others" and should demonstrate a response involving "intense fear, helplessness, or horror". ${ }^{7}$ In earlier versions of the DSM, a cancer diagnosis would not be recognized as a qualifying event; the inclusion of being diagnosed with a life-threatening illness as a qualifying stressful event came after trials for DSM-IV documented higher rates of PTSD in adult cancer survivors than adult controls. ${ }^{61}$

This interpretation was narrowed for DSM-5, which states that "a life-threatening illness or debilitating medical condition is not necessarily considered a traumatic event. Medical incidents that qualify as traumatic events involve sudden, catastrophic events". ${ }^{15}$ The nature of a life-threatening illness such as cancer may not be conducive to identifying a solitary precipitating traumatic event, as the so-called cancer experience encompasses multiple ongoing stressors, many of which occur simultaneously or in close succession. At diagnosis, individuals are confronted with the reality that they will likely die without treatment and they may die with treatment - an event that essentially threatens one's life. Cancer therapy itself imposes abrupt life changes and often involves frequent invasive and painful medical procedures and hospitalization events that threaten one's physical integrity. Approximately two-thirds of all pediatric cancer survivors have at least one complication of their cancer treatment, with one-third of survivors having serious or life-threatening complications. ${ }^{62}$ Late effects manifesting months or years after treatment place survivors at an increased risk of cognitive impairment, second malignant neoplasms, cardiomyopathy, infertility, and a host of other medical and psychosocial sequelae that may also threaten physical integrity. Diagnosis, treatment, and sequelae are usually mutually exclusive events requiring the chronic processing of (traumatic) stress, making it difficult to identify a discrete or episodic stressor. One differential diagnosis suggested by the DSM-IV and DSM-5, adjustment disorder, does account for multiple/continuous stressors such as those associated with chronic illness, but it fails to meet the general profile of survivors presenting with PTSS with regard to the appropriateness, severity, and duration of such distress symptoms. For example, it would be difficult to determine whether marked distress by AYA cancer survivors is out of proportion to the severity or intensity of the stressful event. Additionally, given the longitudinal nature of cancer treatment and sequelae, symptoms are not likely to remit 6 months after the stressor. Another poor fit for this population, acute stress disorder, requires symptoms to cease just 1 month following the stressor. The number of continuous, illness-related stressors associated with survivor PTSS makes cancer-induced PTSS/PTSD a unique pathology perhaps worthy of its own trauma disorder specification.

\section{Mapping DSM criteria onto cancer survivorship}

The challenges associated with measuring posttraumatic stress responses in AYA cancer survivors are not limited to the conceptualization of trauma. Further compromising estimates of posttraumatic stress prevalence among this population is the applicability of the DSM-IV PTSD symptom classification to AYA cancer survivorship. To meet the criteria for a diagnosis of PTSD, an AYA survivor must demonstrate a response to the traumatic event, which is characterized by three symptom clusters (Table 1). In studies where the traumatic event is assumed to be cancer, research procedures may unintentionally prime participating survivors to view their cancer experience as traumatic, thereby creating a "focusing illusion" and risking potential bias in ascertaining prevalence estimates. ${ }^{55}$ Even in cases where the cancer experience is objectively reported as a traumatic stressor, survivors may or may not endorse symptom criteria because of confounding effects of cancer therapy (eg, late effects).

These late effects, commonly associated with cancer survivorship, are not filtered by the diagnostic criteria and have the potential to result in false positives (or false negatives) when assessing prevalence. For example, criterion B requires re-experiencing of the traumatic event via one of the following: intrusive recollections; distressing dreams; reliving the event; or triggered psychological or physiological distress. $^{7}$ It is not unusual for AYA cancer survivors to face realistic risks of relapse and/or other health-compromising morbidities. Re-experiencing or intrusion symptoms may, in their case, reflect well-founded fears about current or future health. ${ }^{21,57}$ Criterion $\mathrm{C}$ requires persistent avoidance of stimuli associated with the trauma and numbing of general responsiveness via at least three of the following: effortful avoidance of thoughts, feeling, or anything associated with the trauma; inability to recall aspects of the trauma; social withdrawal; emotional numbing; and a sense of a foreshortened future. ${ }^{7}$ In the case of AYA cancer survivors, traditional avoidant responses may be rare, as survivors are confronting their health realities regularly through long-term follow-up care. Withdrawal from social interaction and other activities may be more a function of developmental or psychosocial sequelae than psychological avoidance. Diminished recall may have less to do with trauma and may instead represent symptoms of long-term cognitive impairment. ${ }^{21}$ Having a sense of 
foreshortened future may also reflect the very real possibility of relapse rather than a traumatic stress response; unlike other traumatic stressors, cancer is an ongoing event with no clear, temporal end. The final symptom cluster, criterion $\mathrm{D}$, requires persistent, increased arousal via at least two of the following: insomnia; irritability; concentration difficulties; hypervigilance; and an exaggerated startle response. ${ }^{7}$ Distinguishing between common late effects and PTSD symptoms, such as insomnia or delayed sleep onset, and concentration difficulty may prove rather difficult, as these responses also resemble the neurocognitive deficits attributed to cancer therapy. ${ }^{57}$ Criteria $\mathrm{E}$ and $\mathrm{F}$ require that these symptoms persist at least 1 month following exposure to the traumatic event, and that they significantly impair the individual's day-to-day functioning, respectively. Given the nature of cancer's late effects, survivors who experience symptoms are likely to have them persist beyond the 1-month mark. ${ }^{2}$ Similarly, depending on the treatment and late effects endured by survivors, daily functioning may or may not be impaired.

A fourth symptom cluster has been added to the PTSD diagnostic criteria for DSM-5 (Table 3), requiring negative alterations in cognitions and mood associated with the traumatic event. ${ }^{15}$ A diagnosis of PTSD necessitates experiencing at least two of the following symptoms: inability to remember an important aspect of the trauma; persistent and exaggerated negative beliefs about oneself; persistent and disordered cognitions about the cause or consequences of the trauma leading to blame; persistent negative emotional state; diminished interest in activities; interpersonal detachment; and a persistent inability to experience positive emotions. ${ }^{15}$ Although this latest version of the DSM includes a catch-all stipulation (criterion $\mathrm{H}$ ) stating that disturbances must not be attributable to the physiological effects of medication or a medical condition, it is not clear whether late effects of cancer necessarily exempt survivors from symptom endorsement. Given that cancer survivors demonstrate higher levels of cognitive impairment ${ }^{63-68}$ and depressive symptoms than controls, ${ }^{69-73}$ and that a cancer diagnosis can alter one's worldview and challenge existing schemas about the world, looking forward, clinicians, instrument developers, and researchers should take care to disentangle PTSD symptom endorsement from expected, therapy-related effects.

\section{Treatment of PTSS/PTSD}

Evidence-based treatments for PTSD include psychotherapeutic approaches such as cognitive behavior therapy (CBT), as well as pharmacotherapy. ${ }^{74}$ In adults, the treatment literature indicates that effective PTSD interventions involve short-term and cognitive behavioral models. ${ }^{75,76}$ Despite a growing literature base supporting the efficacy of these treatments for PTSD in noncancer populations, a limited number of studies have investigated treatment of PTSS in adolescent survivors of childhood cancer. Stemming from a model that conceptualizes the diagnosis of childhood cancer as a potentially traumatic event, Kazak et $\mathrm{al}^{77}$ developed the Surviving Cancer Competently Intervention Program (SCCIP) for adolescent survivors of childhood cancer and their families. SCCIP, a 1-day, four-session, manualized intervention, uses $\mathrm{CBT}$ and family therapy principles to reduce distress symptoms in survivors. The first two sessions are held separately for survivors, mothers, fathers, and siblings. These sessions provide family members with the opportunity to discuss cancer-related traumatic events, and they utilize cognitive behavioral principles to teach individual coping skills. The third session utilizes multiple family discussion groups to apply information from the first two sessions to the family context. A final session focuses on the application and translation of techniques to the home. Results from a randomized clinical trial of 150 adolescent survivors, their mothers, fathers, and adolescent siblings indicated significant reductions in intrusive thoughts among fathers and arousal symptoms among survivors. ${ }^{78}$ More recently, a pilot study of an Internet-based CBT intervention, Onco-STEP, ${ }^{79}$ resulted in a significant reduction in PTSS and anxiety symptoms among 20 young adult survivors of childhood cancer. The online modules that comprise Onco-STEP utilize an expressive writing technique. A primary aim of this intervention involves reprocessing the traumatic cancer-related event (by writing about the experience), followed by developing coping strategies designed to combat cancer-related fears. Despite the potential promise of these two intervention programs, few empirically supported treatments designed to meet the unique needs of AYA survivors are available.

The concept of posttraumatic growth (PTG) provides an alternate framework for understanding responses to stress that might further inform intervention approaches. PTG, defined as the positive changes or influences resulting from a struggle with a traumatic event, has been reported in some cancer survivors who experience personal growth following the cancer experience. ${ }^{80,81}$ The nature of the relationship between PTG and PTSS has been questioned; one study using a large cohort study suggested a weak but positive relationship between the two constructs. ${ }^{82}$ Further examination of resilience, personal growth, and/or positive change in AYA cancer survivors is critical to understanding the scope of potential trauma responses and how interventions may promote positive outcomes. 


\section{Conclusion}

In sum, AYA cancer survivors appear to be at risk of developing symptoms of posttraumatic stress; however, our ability to quantify the magnitude of this risk is limited by a number of methodological issues present in the current literature. Specifically, prevalence estimates of PTSS/PTSD differ by the assessment measures utilized, the timing of assessment relative to diagnosis, the definition of the outcome, and identification of the precipitating traumatic event. Despite methodological inconsistencies across studies, several static and dynamic risk factors of PTSS have been identified. Empirically supported interventions targeting potentially modifiable risk factors are limited, though CBT approaches have garnered the most support for the treatment of PTSS in AYA survivors of pediatric cancer. Future research is needed to further evaluate existing and forthcoming treatment programs, as well as the applicability of DSM-5 PTSD symptom criteria, to AYA cancer survivors.

\section{Disclosure}

The authors report no conflicts of interest in this work.

\section{References}

1. Ward E, DeSantis C, Robbins A, Kohler B, Jemal A. Childhood and adolescent cancer statistics, 2014. CA Cancer J Clin. 2014;64(2):83-103.

2. Robison LL, Hudson MM. Survivors of childhood and adolescent cancer: life-long risks and responsibilities. Nat Rev Cancer. 2014; 14(1):61-70.

3. Mariotto AB, Rowland JH, Yabroff KR, et al. Long-term survivors of childhood cancers in the United States. Cancer Epidemiol Biomarkers Prev. 2009;18(4):1033-1040.

4. Smith MA, Seibel NL, Altekruse SF, et al. Outcomes for children and adolescents with cancer: challenges for the twenty-first century. J Clin Oncol. 2010;28(15):2625-2634.

5. Howlander N, Noone AM, Krapcho M, et al, editors [webpage on the Internet]. SEER cancer statistics review, 1975-2011. Bethesda, MD National Cancer Institute; 2014. Available from: http://seer.cancer.gov/ csr/1975_2011/. Accessed on October 29, 2014.

6. Bleyer A. Latest estimates of survival rates of the 24 most common cancers in adolescent and young adult Americans. J Adolesc Young Adult Oncol. 2011;1(1):37-42.

7. American Psychiatric Association. Diagnostic and Statistical Manual of Mental Disorders. 4th ed, text revision (DSM-IV-TR). Washington, DC: American Psychiatric Association; 2000.

8. Adolescent and Young Adult Oncology Progress Review Group. Closing the Gap: Research and Care Imperatives for Adolescents and Young Adults with Cancer. Bethesda, MD: US Department of Health and Human Services; 2006.

9. Zebrack B, Isaacson S. Psychosocial care of adolescent and young adult patients with cancer and survivors. J Clin Oncol. 2012;30(11): 1221-1226.

10. Kilpatrick DG, Ruggiero KJ, Acierno R, Saunders BE, Resnick HS, Best CL. Violence and risk of PTSD, major depression, substance abuse/dependence, and comorbidity: results from the National Survey of Adolescents. J Consult Clin Psychol. 2003;71(4):692-700.

11. Merikangas KR, He JP, Burstein M, et al. Lifetime prevalence of mental disorders in US adolescents: results from the National Comorbidity Survey Replication - Adolescent Supplement (NCS-A). J Am Acad Child Adolesc Psychiatry. 2010;49(10):980-989.
12. Kang HK, Natelson BH, Mahan CM, Lee KY, Murphy FM Post-traumatic stress disorder and chronic fatigue syndrome-like illness among GulfWar veterans: a population-based survey of 30,000 veterans. Am J Epidemiol. 2003;157(2):141-148.

13. Galea S, Nandi A, Vlahov D. The epidemiology of post-traumatic stress disorder after disasters. Epidemiol Rev. 2005;27:78-91.

14. Gurevich M, Devins GM, Rodin GM. Stress response syndromes and cancer: conceptual and assessment issues. Psychosomatics. 2002;43(4):259-281.

15. American Psychiatric Association. Diagnostic and Statistical Manual of Mental Disorders. 5th ed (DSM-5). Washington, DC: American Psychiatric Association; 2013

16. Stuber ML, Meeske KA, Krull KR, et al. Prevalence and predictors of posttraumatic stress disorder in adult survivors of childhood cancer. Pediatrics. 2010;125(5):e1124-e1134.

17. Gerhardt CA, Yopp JM, Leininger L, et al. Brief report: post-traumatic stress during emerging adulthood in survivors of pediatric cancer. J Pediatr Psychol. 2007;32(8):1018-1023.

18. Alderfer MA, Navsaria N, Kazak AE. Family functioning and posttraumatic stress disorder in adolescent survivors of childhood cancer. J Fam Psychol. 2009;23(5):717-725.

19. Erickson SJ, Gerstle M, Montague EQ. Repressive adaptive style and self-reported psychological functioning in adolescent cancer survivors. Child Psychiatry Hum Dev. 2008;39(3):247-260.

20. Kazak AE, Alderfer M, Rourke MT, Simms S, Streisand R, Grossman JR. Posttraumatic stress disorder (PTSD) and posttraumatic stress symptoms (PTSS) in families of adolescent childhood cancer survivors. $J$ Pediatr Psychol. 2004;29(3):211-219.

21. Phipps S, Jurbergs N, Long A. Symptoms of post-traumatic stress in children with cancer: does personality trump health status? Psychooncology. 2009;18(9):992-1002.

22. Stuber ML, Kazak AE, Meeske K, et al. Predictors of posttraumatic stress symptoms in childhood cancer survivors. Pediatrics. 1997;100(6): 958-964.

23. Hobbie WL, Stuber M, Meeske K, et al. Symptoms of posttraumatic stress in young adult survivors of childhood cancer. J Clin Oncol. 2000;18(24):4060-4066.

24. Lee YL, Santacroce SJ. Posttraumatic stress in long-term young adult survivors of childhood cancer: a questionnaire survey. Int J Nurs Stud. 2007;44(8):1406-1417.

25. Meeske KA, Ruccione K, Globe DR, Stuber ML. Posttraumatic stress, quality of life, and psychological distress in young adult survivors of childhood cancer. Oncol Nurs Forum. 2001;28(3):481-489.

26. Rourke MT, Hobbie WL, Schwartz L, Kazak AE. Posttraumatic stress disorder (PTSD) in young adult survivors of childhood cancer. Pediatr Blood Cancer. 2007;49(2):177-182.

27. Santacroce SJ, Lee YL. Uncertainty, posttraumatic stress, and health behavior in young adult childhood cancer survivors. Nurs Res. 2006;55(4):259-266.

28. Stuber ML, Meeske KA, Leisenring W, et al. Defining medical posttraumatic stress among young adult survivors in the Childhood Cancer Survivor Study. Gen Hosp Psychiatry. 2011;33(4):347-353.

29. Varela VS, Ng A, Mauch P, Recklitis CJ. Posttraumatic stress disorder (PTSD) in survivors of Hodgkin's lymphoma: prevalence of PTSD and partial PTSD compared with sibling controls. Psychooncology. 2013;22(2):434-440.

30. Wiener L, Battles H, Bernstein D, et al. Persistent psychological distress in long-term survivors of pediatric sarcoma: the experience at a single institution. Psychooncology. 2006;15(10):898-910.

31. Brown RT, Madan-Swain A, Lambert R. Posttraumatic stress symptoms in adolescent survivors of childhood cancer and their mothers. JTrauma Stress. 2003;16(4):309-318.

32. Erickson SJ, Steiner H. Trauma and personality correlates in long-term pediatric cancer survivors. Child Psychiatry Hum Dev. 2001;31(3): 195-213.

33. Kazak AE, Barakat LP, Alderfer M, et al. Posttraumatic stress in survivors of childhood cancer and mothers: development and validation of the Impact of Traumatic Stressors Interview Schedule. J Clin Psychol Med Settings. 2001;8(4):307-323. 
34. Kazak AE, Derosa BW, Schwartz LA, et al. Psychological outcomes and health beliefs in adolescent and young adult survivors of childhood cancer and controls. J Clin Oncol. 2010;28(12):2002-2007.

35. Kwak M, Zebrack BJ, Meeske KA, et al. Prevalence and predictors of post-traumatic stress symptoms in adolescent and young adult cancer survivors: a 1-year follow-up study. Psychooncology. 2013;22(8): 1798-1806.

36. Pelcovitz D, Libov BG, Mandel F, Kaplan S, Weinblatt M, Septimus A. Posttraumatic stress disorder and family functioning in adolescent cancer. J Trauma Stress. 1998;11(2):205-221.

37. Schrag NM, McKeown RE, Jackson KL, Cuffe SP, Neuberg RW. Stressrelated mental disorders in childhood cancer survivors. Pediatr Blood Cancer. 2008;50(1):98-103.

38. Schwartz LA, Kazak AE, Derosa BW, Hocking MC, Hobbie WL, Ginsberg JP. The role of beliefs in the relationship between health problems and posttraumatic stress in adolescent and young adult cancer survivors. J Clin Psychol Med Settings. 2012;19(2):138-146.

39. Armstrong JG, Putnam FW, Carlson EB, Libero DZ, Smith SR. Development and validation of a measure of adolescent dissociation: the Adolescent Dissociative Experiences Scale. J Nerv Ment Dis. 1997;185(8):491-497.

40. Foa EB, Cashman L, Jaycox L, Perry K. The validation of a self-report measure of posttraumatic stress disorder: the Posttraumatic Diagnostic Scale. Psychol Assess. 1997;9(4):445-451.

41. Pynoos R, Rodriguez N, Steinberg A, Stuber M, Frederick C. UCLA PTSD Index for DSM-IV: Adolescent Version. Los Angeles, CA: UCLA Trauma Psychiatry Service; 1998.

42. Weathers FW, Ford J. Psychometric review of PTSD checklist (PCLC, PCL-S, PCL-M, PCL-PR). In: Stamm BH, editor. Measurements of Stress, Trauma, and Adaptation. Lutherville, MD: Sidran Press; 1996:250-252.

43. Rodriguez N, Steinberg A, Pynoos RS. The Child Posttraumatic Stress Reaction Index, Revision 2. Los Angeles, CA: UCLA Trauma Psychiatry Program. 2001.

44. Briere J. Trauma Symptom Checklist for Children: Professional Manual. Odessa, FL: Psychological Assessment Resources Inc.; 1996.

45. Orvaschel H. Schedule for Affective Disorders and Schizophrenia for School-Age Children - Epidemiologic Version - 5 (K-SADS-E-S). Fort Lauderdale, FL: Nova Southeast University; 1995.

46. First MB, Spitzer RL, Gibbon M, Williams JB. Structured Clinical Interview for DSM-IV Axis I Disorders-Nonpatient Edition (SCID-I/NP, Version 2.0). New York, NY: New York State Psychiatric Institute; 1995.

47. Newman E, Weathers F, Nader K, Kalouped D, Pynoos R, Blake D. Clinician-Administered PTSD Scale for Children and Adolescents (CAPS-CA). Los Angeles, CA: Western Psychological Services; 2004.

48. Davidson J, Smith R, Kudler H. Validity and reliability of the DSM-III criteria for posttraumatic stress disorder. Experience with a structured interview. J Nerv Ment Dis. 1989;177(6):336-341.

49. Fisher PW, Shaffer D, Piacentini JC, et al. Sensitivity of the Diagnostic Interview Schedule for Children, 2nd edition (DISC-2.1) for specific diagnoses of children and adolescents. J Am Acad Child Adolesc Psychiatry. 1993;32(3):666-673.

50. Derogatis LR, Melisaratos N. The Brief Symptom Inventory: an introductory report. Psychol Med. 1983;13(3):595-605.

51. Horowitz M, Wilner N, Alvarez W. Impact of Event Scale: a measure of subjective stress. Psychosom Med. 1979;41(3):209-218.

52. Ware JE, Snow KK, Kosinski M, Gandek B. SF-36 Health Survey: Manual and Interpretation Guide. Boston, MA: The Health Institute, New England Medical Center; 1993.

53. Ware J Jr, Kosinski M, Keller SD. A 12-Item Short-Form Health Survey: construction of scales and preliminary tests of reliability and validity. Med Care. 1996;34(3):220-233.

54. Endicott J, Spitzer RL, Fleiss JL, Cohen J. The global assessment scale. A procedure for measuring overall severity of psychiatric disturbance. Arch Gen Psychiatry. 1976;33(6):766-771.
55. Phipps S, Klosky JL, Long A, et al. Posttraumatic stress and psychological growth in children with cancer: has the traumatic impact of cancer been overestimated? J Clin Oncol. 2014;32(7):641-646.

56. Kangas M, Henry JL, Bryant RA. Posttraumatic stress disorder following cancer. A conceptual and empirical review. Clin Psychol Rev. 2002;22(4):499-524.

57. Bruce M. A systematic and conceptual review of posttraumatic stress in childhood cancer survivors and their parents. Clin Psychol Rev. 2006;26(3):233-256.

58. Yehuda R, McFarlane AC. Conflict between current knowledge about posttraumatic stress disorder and its original conceptual basis. Am J Psychiatry. 1995;152(12):1705-1713.

59. Davis L, Siegel LJ. Posttraumatic stress disorder in children and adolescents: a review and analysis. Clin Child Fam Psychol Rev. 2000;3(3):135-154.

60. Tedstone JE, Tarrier N. Posttraumatic stress disorder following medical illness and treatment. Clin Psychol Rev. 2003;23(3):409-448.

61. Rourke MT, Stuber ML, Hobbie WL, Kazak AE. Posttraumatic stress disorder: understanding the psychosocial impact of surviving childhood cancer into young adulthood. J Pediatr Oncol Nurs. 1999;16(3): 126-135.

62. Oeffinger KC, Hudson MM. Long-term complications following childhood and adolescent cancer: foundations for providing risk-based health care for survivors. CA Cancer J Clin. 2004;54(4):208-236.

63. Armstrong GT, Reddick WE, Petersen RC, et al. Evaluation of memory impairment in aging adult survivors of childhood acute lymphoblastic leukemia treated with cranial radiotherapy. J Natl Cancer Inst. 2013;105(12):899-907.

64. Kadan-Lottick NS, Zeltzer LK, Liu Q, et al. Neurocognitive functioning in adult survivors of childhood non-central nervous system cancers. J Natl Cancer Inst. 2010;102(12):881-893.

65. Krull KR, Brinkman TM, Li C, et al. Neurocognitive outcomes decades after treatment for childhood acute lymphoblastic leukemia: a report from the St Jude lifetime cohort study. J Clin Oncol. 2013;31(35): 4407-4415.

66. Krull KR, Sabin ND, Reddick WE, et al. Neurocognitive function and CNS integrity in adult survivors of childhood hodgkin lymphoma. J Clin Oncol. 2012;30(29):3618-3624.

67. Krull KR, Zhang N, Santucci A, et al. Long-term decline in intelligence among adult survivors of childhood acute lymphoblastic leukemia treated with cranial radiation. Blood. 2013;122(4):550-553.

68. Mulhern RK, Merchant TE, Gajjar A, Reddick WE, Kun LE. Late neurocognitive sequelae in survivors of brain tumours in childhood. Lancet Oncol. 2004;5(7):399-408.

69. Hudson MM, Mertens AC, Yasui Y, et al; Childhood Cancer Survivor Study Investigators. Health status of adult long-term survivors of childhood cancer: a report from the Childhood Cancer Survivor Study. JAMA. 2003;290(12):1583-1592.

70. Schultz KA, Ness KK, Whitton J, et al. Behavioral and social outcomes in adolescent survivors of childhood cancer: a report from the childhood cancer survivor study. J Clin Oncol. 2007;25(24):3649-3656.

71. Zebrack BJ, Gurney JG, Oeffinger K, et al. Psychological outcomes in long-term survivors of childhood brain cancer: a report from the childhood cancer survivor study. J Clin Oncol. 2004;22(6):999-1006.

72. Zebrack BJ, Zeltzer LK, Whitton J, et al. Psychological outcomes in long-term survivors of childhood leukemia, Hodgkin's disease, and non-Hodgkin's lymphoma: a report from the Childhood Cancer Survivor Study. Pediatrics. 2002;110(1 Pt 1):42-52.

73. Zeltzer LK, Lu Q, Leisenring W, et al. Psychosocial outcomes and health-related quality of life in adult childhood cancer survivors: a report from the childhood cancer survivor study. Cancer Epidemiol Biomarkers Prev. 2008;17(2):435-446.

74. Iribarren J, Prolo P, Neagos N, Chiappelli F. Post-traumatic stress disorder: evidence-based research for the third millennium. Evid Based Complement Alternat Med. 2005;2(4):503-512.

75. Kar N. Cognitive behavioral therapy for the treatment of post-traumatic stress disorder: a review. Neuropsychiatr Dis Treat. 2001;7:167-181. 
76. Keane T. Psychological and behavioral treatments of post-traumatic stress disorder. In: Nathan P, Gorman J, editors. A guide to treatments that work. New York: Oxford University Press; 1998.

77. Kazak AE, Simms S, Barakat L, et al. Surviving cancer competently intervention program (SCCIP): a cognitive-behavioral and family therapy intervention for adolescent survivors of childhood cancer and their families. Fam Process. 1999;38(2):175-191.

78. Kazak AE, Alderfer MA, Streisand R, et al. Treatment of posttraumatic stress symptoms in adolescent survivors of childhood cancer and their families: a randomized clinical trial. J Fam Psychol. 2004;18(3): 493-504.

79. Seitz DC, Knaevelsrud C, Duran G, Waadt S, Loos S, Goldbeck L. Efficacy of an internet-based cognitive-behavioral intervention for long-term survivors of pediatric cancer: a pilot study. Support Care Cancer. 2014;22(8):2075-2083.
80. Barakat LP, Alderfer MA, Kazak AE. Posttraumatic growth in adolescent survivors of cancer and their mothers and fathers. J Pediatr Psychol. 2006;31(4):413-419.

81. Duran B. Posttraumatic growth as experienced by childhood cancer survivors and their families: a narrative synthesis of qualitative and quantitative research. J Pediatr Oncol Nurs. 2013;30(4):179-197.

82. Klosky JL, Krull KR, Kawashima T, et al. Relations between posttraumatic stress and posttraumatic growth in long-term survivors of childhood cancer: a report from the Childhood Cancer Survivor Study. Health Psychol. 2014;33(8):878-882.

\section{Publish your work in this journal}

Clinical Oncology in Adolescents and Young Adults is an international, peer-reviewed, open access journal publishing original research, reports, editorials, reviews and commentaries on all aspects of epidemiology, diagnosis and treatment of cancers in adolescents and young adults. The manuscript management system is completely online and includes a very quick and fair peer-review system. Visit http://www.dovepress.com/testimonials.php to read real quotes from published authors.

\footnotetext{
Submit your manuscript here: http://www.dovepress.com/clinical-oncology-in-adolescents-and-young-adults-journal
} 